\section{Adjuvante Therapie beim Pankreaskarzinom hilft auch bei verzögertem Beginn}

\begin{abstract}
Die Resektion eines Pankreaskarzinoms geht mit hoher Morbidität und vielen Komplikationen einher, was oft den Beginn der adjuvanten Chemotherapie verzögert. Welchen Einfluss hat dies auf das Gesamtüberleben (OS)?
\end{abstract}

A us der US-amerikanischen National Cancer Data Base (NCDB) wurden die Daten von 6.706 Pankreaskarzinompatienten (Stadium I-III) selektiert, die sich in den Jahren 2003 bis 2011 einer Tumorresektion mit anschließender Chemotherapie unterzogen hatten. In der Analyse wurde differenziert zwischen dem Beginn der Chemotherapie innerhalb von 12 Wochen nach der Resektion $(n=3.723)$ oder danach $(n=669)$.

Ein späterer Beginn der Chemotherapie war assoziiert mit höherem Alter und mehr Komorbiditäten. Auch die Art der Operation war relevant: Bei Whipple-Resektion wie auch bei totaler Pankreatektomie war das Risiko für einen späteren Beginn der adjuvanten Chemo- therapie höher als bei distaler Resektion. Die adjuvante Chemotherapie ging insgesamt mit einem klaren Überlebensvorteil gegenüber einer alleinigen Resektion einher $(p<0,0001)$. Dabei war es nicht wesentlich, wann sie begonnen worden war. Bei ausschließlicher Operation lag das mediane OS bei 14,4 Monaten, bei Beginn der Chemotherapie in den ersten 12 Wochen bei 22 Monaten, bei späterem Beginn bei 20,8 Monaten ( $p=0,5301$ bei Vergleich der adjuvanten Kohorten). Auch stratifiziert nach der Pathologie zeigte sich kein Unterschied im OS-Gewinn bei frühem und spätem Beginn der adjuvanten Therapie.

In der multivariaten Analyse wurden Patienten-, Erkrankungs- und Operati- onscharakteristika mit berücksichtigt. Auch hier zeigte sich ein signifikanter Vorteil für eine adjuvante Chemotherapie sowohl im Falle des frühen als auch des verzögerten Beginns. Die Mortalitätsrate war insgesamt gegenüber der Resektion alleine um $35 \%$ verringert ( $p<0,0001$ für beide Strategien verglichen mit alleiniger Resektion).

Fazit: Der Beginn der adjuvanten Chemotherapie innerhalb von 12 Wochen nach der chirurgischen Therapie eines Pankreaskarzinoms geht nicht mit einem signifikanten Überlebensvorteil gegenüber einer später begonnenen adjuvanten Chemotherapie einher. Auch wenn sich durch Komplikationen oder eine längere Erholungsphase nach der Operation der Beginn der Adjuvanz verzögert, sollte diese angeboten werden, da sie immer noch einen Überlebensvorteil bedeutet.

Friederike Klein

Mirkin KA et al. Time to the initiation of adjuvant chemotherapy does not impact survival in patients with resected pancreatic cancer. Cancer. 2016;122(19):2979-87.

\title{
pNET: Abschließende Analyse für mTOR-Inhibitor
}

\author{
Bei pankreatischen neuroendokrinen Tumoren (pNET) hatte die Therapie \\ mit Everolimus in der RADIANT-3-Studie das mediane progressionsfreie \\ Überleben gegenüber Placebo um 6,4 Monate verlängert. Jetzt liegt die \\ finale Analyse zum Gesamtüberleben (OS) vor.
}

m Rahmen der Studie hatten Patienten mit fortgeschrittenem, progredientem pNET niedrigen oder intermediären Grads randomisiert $10 \mathrm{mg} /$ Tag Everolimus $(n=207)$ oder Placebo $(n=203)$ erhalten. Bei Krankheitsprogress war ein Crossover aus dem Placebo- in den aktiven Arm erlaubt. Zudem konnte die Everolimus-Therapie nach Ende der verblindeten Studienphase weitergeführt werden. Als mögliche für das OS relevante Biomarker wurden die Basiswerte von Chromogranin A (CgA), der Neuronspezifischen Enolase (NSE) und verschiedener löslicher angiogener Biomarker untersucht. 225 Patienten erhielten Everolimus in der offenen Extensionsphase der Studie, davon 172 Patienten (85\%) des ursprünglichen Placebo-Arms und 53 (26\%) des Everolimus-Arms.

Das mediane OS betrug im Everolimus-Arm 44,0 Monate (95\%-Konfidenzintervall [95\%-KI] 35,6-51,8 Monate), im Placeboarm 37,7 Monate (95\%-KI 29,1-45,8 Monate). Der Unterschied von 6,3 Monaten war nicht statistisch signifikant (Hazard Ratio [HR] 0,94, $95 \%$-KI $0,73-1,20 ; p=0,30)$. Das führen die Forscher um James C. Yao vor allem auf die sehr hohe Cross-over-Rate vom Placeboin den Everolimus-Arm zurück.

Zum finalen Auswertungszeitpunkt waren 256 Patienten $(62,4 \%)$ verstorben, $126(60,9 \%)$ aus dem ursprünglichen Everolimus- und 130 (64,0\%) aus dem ursprünglichen Placeboarm. Mit einer schlechten OS-Prognose assoziiert waren erhöhte Werte von CgA, NSE, PLGF („placenta growth factor") und dem löslichen Typ-1-Rezeptor für VEGF („vascular endothelial growth factor") bei Studienaufnahme.

Die Nebenwirkungen der EverolimusTherapie entsprachen den von dem mTOR-Inhibitor bekannten, darunter Stomatitis, Hautausschläge und Diarrhö.

Fazit: Die Therapie mit Everolimus war mit einem medianen OS von 44 Monaten assoziiert - das ist länger als bisher je in einer Phase-III-Studie beim fortgeschrittenen pNET berichtet. Die Randomisierung zur Everolimus-Therapie resultierte in einem 6,3-monatigen OSVorteil, der - wahrscheinlich wegen des hohen Crossovers - nicht statistisch signifikant war.

Friederike Klein

Yao JC et al. Everolimus for the Treatment of Advanced Pancreatic Neuroendocrine Tumors:

Overall Survival and Circulating Biomarkers From the Randomized, Phase III RADIANT-3 Study. J Clin Oncol. 2016 Sep 12. [Epub ahead of print]. 